\title{
The metabolic demands of swimming behavior influence the evolution of skeletal muscle fiber design in the brachyuran crab family Portunidae
}

\author{
Kristin M. Hardy $\cdot$ Sean C. Lema $\cdot$ Stephen T. Kinsey
}

\begin{abstract}
We investigated the influence of intracellular diffusion on muscle fiber design in several swimming and non-swimming brachyuran crabs. Species with sustained swimming behavior had aerobic dark fibers subdivided into small metabolic functional units, creating short diffusion distances necessary to support the high rates of aerobic ATP turnover associated with endurance activity. This dark fiber design was observed in all swimming species including Ovalipes ocellatus, which has apparently evolved swimming behavior independently of other Portunidae. In addition, we observed fiber and subdivision size-dependent differences in organelle distribution. Mitochondria, which rely on oxygen to function, were uniformly distributed in small fibers/subdivisions, but were clustered at the fiber periphery in larger fibers. The inverse pattern was observed for nuclei, which are not oxygen dependent, but rely on the transport of slow diffusing macromolecules. Phylogenetically independent contrast analysis revealed that these relationships were largely independent of phylogeny. Our results demonstrate cellular responses to diffusion that were necessary for the evolution of swimming and that are likely to be broadly applicable.
\end{abstract}

\section{Introduction}

The basal muscles that power swimming in the blue crab, Callinectes sapidus, grow hypertrophically during postmetamorphic development, so that fiber diameters increase from $<60 \mu \mathrm{m}$ in juveniles to $>600 \mu \mathrm{m}$ in adults (Boyle et al. 2003). At these fiber sizes, intracellular diffusion distances begin to limit certain processes critical to normal muscle cell function (Hardy et al. 2006; Kinsey et al. 2005). Potential limitations caused by excessive intracellular diffusion distances have long been hypothesized as one of the primary reasons that cells are relatively small (typically within an order of magnitude of $10 \mu \mathrm{m}$ ) (Koch 1996; Russell et al. 2000; Teissier 1939; Thompson 1917). Rapid intracellular diffusion of oxygen and small metabolites [e.g., ATP, arginine phosphate (AP) and inorganic phosphate $\left(\mathrm{P}_{\mathrm{i}}\right)$ ] is critical to maintaining high rates of aerobic metabolism (Kim et al. 1998; Mainwood and Rakusan 1982), and the diffusion of larger RNA and polypeptide molecules is important for protein turnover (Fusco et al. 2004; Russell and Dix 1992). Thus, diffusion may provide selective pressure for the evolution of basic cellular design and function, and this role may become greater as intracellular diffusion distances and/or aerobic metabolic rates become greater.

Basal swimming muscles are composed primarily of two fiber types: light fibers that power anaerobic burst swimming and dark fibers that power aerobic endurance swimming (Tse et al. 1983). The light fibers rely on maximal rates of aerobic metabolism only during post-contractile recovery, which is associated with low ATP turnover rates, while the dark fibers use aerobic processes to support the high rates of ATP turnover associated with sustained contractile activity. We previously demonstrated that diffusion has a considerable impact on cellular organization in the basal swimming muscles of the blue crab and that these 
effects were distinctly different between the anaerobic light fibers and aerobic dark fibers as a result of their fundamentally different metabolic requirements (Boyle et al. 2003; Hardy et al. 2009; Johnson et al. 2004).

During hypertrophic growth, the anaerobic light fibers of C. sapidus appear to maintain cellular function by redistributing certain organelles in a way that minimizes intracellular diffusive path lengths. Mitochondria, for example, are homogenously scattered throughout each fiber in juvenile animals so that there are nearly equal numbers of mitochondria in the fiber interior (intermyofibrillar or IM mitochondria) and at the fiber periphery (subsarcolemmal or SS mitochondria). However, during growth, mitochondria begin to cluster near the sarcolemma, and in adults, virtually no mitochondria occur within the fiber core (Boyle et al. 2003; Hardy et al. 2009). This rearrangement effectively reduces transport distances for oxygen from the blood to the mitochondria, but increases travel distances for small, rapidly diffusing metabolites like ATP and AP.

Muscle fibers are multi-nucleated cells, and myonuclear distribution also changes during hypertrophic growth, but in a pattern that is the inverse of that seen for mitochondria. In small juvenile fibers, myonuclei are located exclusively at the fiber periphery adjacent to the sarcolemmal membrane (the characteristic pattern in vertebrate fibers), but during fiber growth, nuclei begin to occupy more centrally located positions within the fiber as well. This shift results in reduced intracellular transport distances for the large, slowly diffusing protein and RNA products required by the fiber for turnover of metabolic and contractile machinery (Hardy et al. 2009).

The aerobic dark fibers, on the other hand, have to meet much higher ATP demands during steady-state contraction, and high reaction rates can result in a diffusion limiting environment even in fibers with small diameters. To satisfy the opposing demands for hypertrophic growth and short diffusion path lengths, the dark fibers have developed a network of highly perfused, mitochondria-rich subdivisions (Johnson et al. 2004; Tse et al. 1983) that increase in number while maintaining a constant size $(\sim 35 \mu \mathrm{m})$ during growth (Johnson et al. 2004). In this way, the aerobic fibers preserve an effective metabolic diameter throughout development that is well within the range of cellular dimensions typical of aerobic muscle from other animals. The perfused subdivisions result in greatly reduced diffusion distances and increased oxygen availability to the mitochondria. As such, nuclei and mitochondria do not undergo the ontogenetic shift in organelle distribution observed in anaerobic fibers. In both adult and juvenile animals, nuclei are found exclusively at the subdivision periphery, while mitochondria are found predominantly at the periphery of each subdivision and, at a lower density, between the myofibrils. Reaction-diffusion mathematical models demonstrated that the ontogenetic shifts in both organelle distribution in the light fibers and the subdivision of dark fibers are essential to promote observed rates of aerobic metabolism in C. sapidus muscle (Hardy et al. 2009).

Organelle distribution in adult skeletal muscle fibers is a plastic property. Mitochondrial distribution and morphology have been shown to vary dramatically in response to factors including temperature (Tyler and Sidell 1984), hypoxia (Hoppeler and Vogt 2001) and exercise (Chilibeck et al. 2002; Howald et al. 1985; Kayar et al. 1986). Likewise, nuclei have been reported to realign themselves with newly formed blood vessels in skeletal muscle fibers subject to chronic stimulation following denervation (Ralston et al. 2006). The processes by which organelles migrate and anchor inside of cells have been studied extensively (Bitoun et al. 2005; Frederick and Shaw 2007; Milner et al. 1996; Ralston et al. 2006; Rube and van der Bliek 2004; Smirnova et al. 1998; Starr 2007; Starr and Han 2002). However, current understanding of the mechanisms that regulate organelle movement is limited, and the signals that result in the relocation of mitochondria or nuclei within an adult (or embryonic) muscle fiber are largely unknown.

There are many potential regulatory mechanisms that could dictate the intracellular arrangement of organelles, and these strategies are not necessarily mutually exclusive. The distribution of organelles within a cell may simply be a product of phylogenetic inertia. This term refers to the stability of a trait that results from the influence of an ancestor on its descendant (for review see Blomberg and Garland 2002). If there is no selective pressure to modify the placement of organelles in a fiber, then an organism will likely share the same distribution as their ancestor. Alternatively, intracellular organelle distribution may be the product of a genetic developmental program (Badrinath and White 2003; van Blerkom 1991). A cell must be able to function over the entire range of sizes it will span in its lifetime. If the terminal cell size or degree of expected hypertrophy is encoded in the genome of an animal, then certain mechanisms may be implemented early in development to prepare each cell for constraints that will surface only after substantial growth has occurred. A third possibility is that the cellular organization of organelles is the direct and immediate product of some prevailing intracellular condition-in particular, diffusion constraints. For example, mitochondrial distribution could be responsive to intracellular oxygen concentrations. During hypertrophic growth, oxygen gradients across the cell may steepen due to increasing fiber size, and mitochondria may shift from areas of low to high oxygen concentration to maintain rates of oxidative phosphorylation sufficient to preserve function in that fiber.

The aim of the present study was to investigate cellular organization across a range of fiber sizes spanned by adults of several related, but morphologically and behaviorally 
divergent, species of Portunid and non-Portunid crabs to examine the relative effects of diffusion and shared common ancestry on fiber design. The family Portunidae comprises a group of brachyuran crabs well known for their swimming abilities (Fiedler 1930; Judy and Dudley 1970; Spirito 1972). Portunids exhibit a number of characteristic morphological adaptations that have facilitated the evolution of swimming behavior. Most notably, the 5th pereiopods have been modified into flattened, oar-like paddles, and the carapace has been laterally extended and dorsoventrally compressed to increase hydrodynamic efficiency during sideways swimming (Hartnoll 1971). The basal swimming musculature in particularly adept swimming portunids is also generally enlarged and exhibits severe fiber hypertrophy, most likely to fulfill the high power requirements of swimming (Cochran 1935).

Within the portunid family, however, there is considerable variation in the extent of these specializations and hence the range of swimming proficiency. In some species, swimming behaviors only occur during brief feeding or escape events, while other species have adopted an entirely pelagic lifestyle (Hartnoll 1971). Carcinus maenus, for example, is particularly interesting because it is one of the only portunids whose 5 th pereiopods have retained their original walking leg characteristics. As such, C. maenus is a much weaker swimmer than many of the other more modified portunids. Although this family is popularly referred to as "swimming crabs," there are representative species from at least 12 other brachyuran families that also exhibit some capacity to swim (reviewed in Hartnoll 1971).

Previously, we documented changes in cellular organization that occurred during ontogenetic muscle fiber hypertrophy in a single Portunid species, C. sapidus (Hardy et al. 2009). From this study, it could not be conclusively determined whether the observed changes in cellular organization were true adaptive responses to changing diffusion constraints associated with swimming behavior. In the current study, we examined the homologous basal muscles from eight different brachyuran crab species (six portunids and two non-portunids) —all in their mature adult form-and measured fiber/subdivision size, as well as mitochondrial and nuclear density and distribution in anaerobic light fibers and aerobic dark fibers. Using 16S rDNA sequences, we generated a phylogeny for these species from which we performed a phylogenetically independent contrast (PIC) analysis (Felsenstein 1985). The PIC analysis determines whether an observed trait is the product of phylogenetic inertia (shared common ancestry), whereas a trait that is found to be independent of phylogeny can be considered an evolutionary adaptation. We used this analysis to discern the relative influence of phylogenetic ancestry and intracellular diffusion limitations on organelle distribution. We hypothesized that all portunids evolved the ability to swim aerobically by subdividing the dark fibers, and that higher aerobic swimming capacity is associated with smaller subdivisions and higher mitochondrial densities. Additionally, we hypothesized that the patterns in cellular design we previously observed during growth in $C$. sapidus light and dark fibers would be broadly observable across a range of portunid (and non-portunid) species, and that these patterns would be independent of phylogeny. Such an independence from phylogeny would provide further evidence that intracellular organelle distribution is, in fact, an adaptation to prevailing intracellular diffusion conditions.

\section{Materials and methods}

Animals

Six species from the swimming crab family, Portunidae, were included in this study: Callinectes sapidus, Portunus sayi, Portunus gibbesii, Portunus spinimanus, Carcinus maenus and Ovalipes ocellatus. These species were chosen for their wide range of swimming abilities, appendage modifications and average adult sizes (as described by Hartnoll 1971 and Williams 1974). Animals were also chosen with regard to their phylogenetic relationships, which were recently resolved from $16 \mathrm{~S}$ rRNA gene sequences for many species of Portunids (Mantelatto et al. 2007; Robles et al. 2007). In addition, Cancer magister (F. Cancridae) and Menippe mercenaria (F. Xanthidae) were included in this study as representative non-portunid, non-swimming crabs. Animals were obtained locally (Wilmington, NC, USA) from inshore sweep netting, offshore trawling and commercial fisherman, as well as purchased live from national commercial and marine organism suppliers (Woods Hole Marine Biological Laboratories and Gulf Specimens Marine Laboratories). Species identification, when necessary, was verified on the basis of morphological characteristics (Rathbun 1930; Williams 1974) and only mature, adult and intermoult animals were used, with no preference given to sex. Animals were maintained in full-strength, filtered seawater (35\% salinity, $\left.21^{\circ} \mathrm{C}\right)$ in aerated, recirculating aquariums, though in most cases, they were processed immediately upon arrival and did not require long term housing. Prior to use in all experiments, animals were sexed, and their carapace width and body mass were measured.

\section{Dissection}

Crabs were first rapidly cut through the cerebral ganglion, and then the dorsal carapace, heart, reproductive and digestive organs were removed. The gills and other supporting architecture were cut off to expose the basal cavity, 
which houses the basal muscles of the 5th pereiopods (remoter, promoter, levator, depressor) (Cochran 1935). The muscle group that originates on the median plate and inserts at the large tendon of the 5th pereiopods, which powers swimming (or walking) movement in the final pair of legs, was carefully isolated and removed from each animal.

Cellular dimensions and mitochondrial density

After being removed from the animal, the muscle $(N=3$ per species) was subsequently frozen in isopentane cooled in liquid nitrogen, mounted in optimal cutting temperature (OCT) compound and frozen again in cooled isopentane. Samples were equilibrated to $-18^{\circ} \mathrm{C}$ and sectioned immediately. Muscle cross sections were obtained on a ReichertJung Leica Cryocut 1800 microtome (Leica Microsystems; Wetzler, Germany) at $30 \mu \mathrm{m}$ thickness using a systematic random sampling method to ensure complete representation of mitochondrial and nuclear distribution throughout the muscle (Howard and Reed 1998). Sections were picked up on room temperature Superfrost PLUS slides (12-550-15; Fisher Scientific) and allowed to air-dry for $30 \mathrm{~min}$ at room temperature. Slides were then incubated in a succinic dehydrogenase (SDH)-staining solution $[12.5 \%$ solution of nitro blue tetrazolium (NBT) in equal volumes of $0.2 \mathrm{M}$ sodium succinate and $0.2 \mathrm{M}$ phosphate buffer ( $\mathrm{pH}$ 7.6)] (Presnell and Schreibman 1997) at $37^{\circ} \mathrm{C}$ for $1 \mathrm{~h}$ while gently agitating every $5 \mathrm{~min}$. Muscle fibers with a high oxidative capacity, and hence high mitochondrial content, had increased SDH staining. At this incubation duration, the high resolution of SDH staining allowed individual mitochondria and mitochondrial clusters to be distinguished. After incubation, slides were rinsed in phosphate-buffered saline (PBS) for $1 \mathrm{~min}$ at room temperature and then fixed for $1 \mathrm{~h}$ in a $10 \%$ formalin $10 \% \mathrm{NaCl}$ solution. Slides were dehydrated in a graded ethanol series $(70,95,100 \%$ for $3 \mathrm{~min}$ each), cleared in toluene for $3 \mathrm{~min}$, and mounted with Permount.

Stained sections were viewed using an Olympus BH-2 light microscope, and digital images were captured using a Diagnostic Images, Inc. Spot RT camera. Fiber and subdivision margins were traced using Adobe Photoshop v7.0, and resultant polygons were analyzed with Image Pro Plus (IPP) v4.1.0.9 to calculate fiber and subdivision crosssectional area (CSA), circumference and mean diameter. To determine relative mitochondrial densities, intensity profiles were collected from images of both light fibers and dark fiber subdivisions. Using IPP, a straight, one pixelwide line was drawn randomly across the entire width of an individual fiber/subdivision cross section (Fig. 1a), and a relative intensity value (on a scale from 0 to 255 , where 0 is pure white and 255 is pure black) was assigned to each (a)

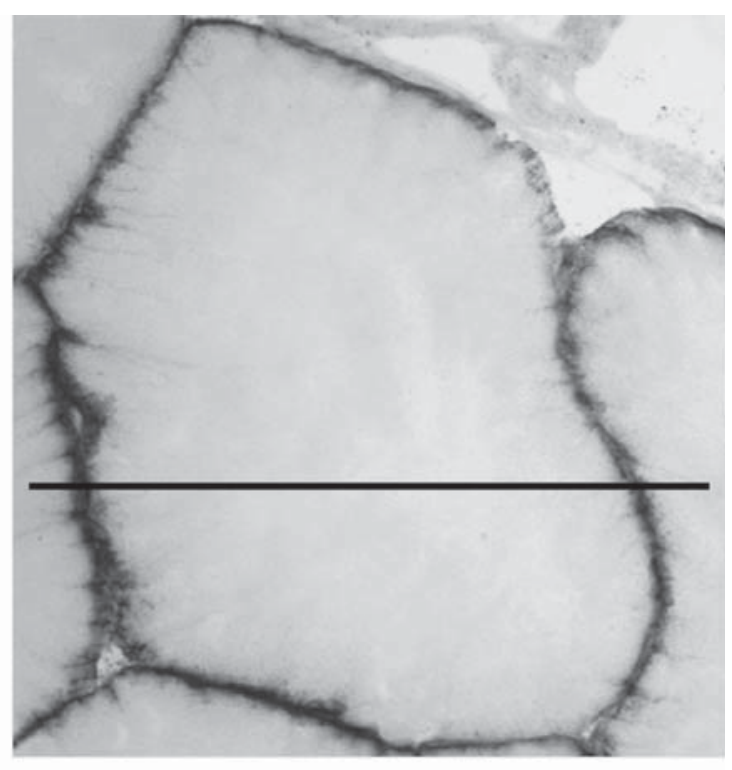

(b)
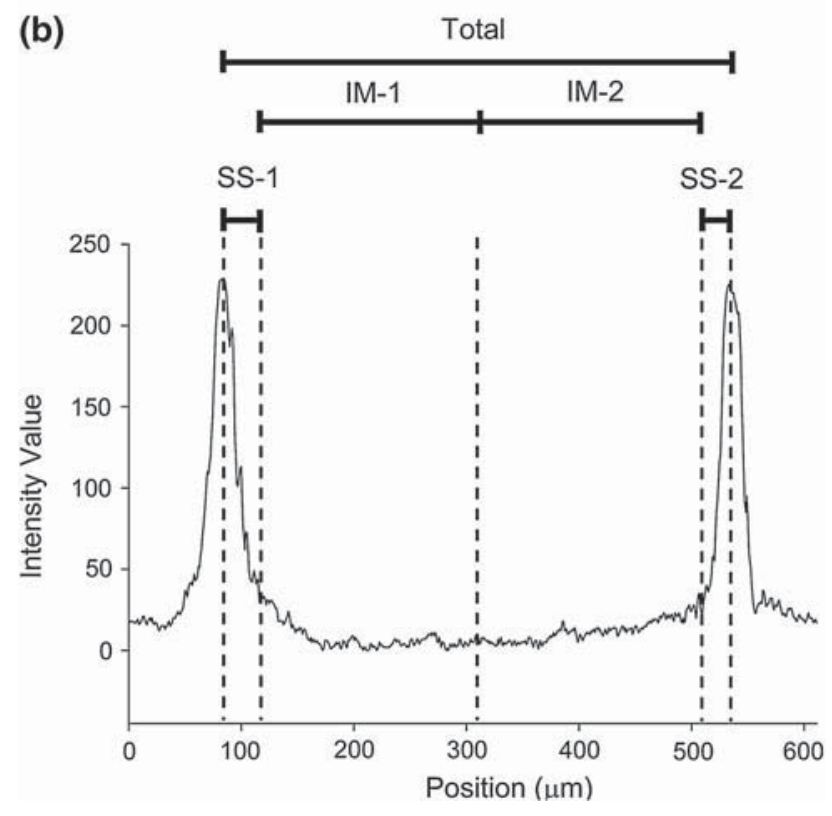

Fig. 1 Method of estimating mitochondrial density from intensity profiles of muscle cross sections stained for succinic dehydrogenase (SDH) activity. a Representative image of an anaerobic light fiber (here from M. mercenaria) that has been stained for SDH. A horizontal line is placed across the diameter of an entire fiber, and an intensity value (from 0 to 255) is calculated by the IPP software for each pixel of the image crossed by the line. $\mathbf{b}$ The intensity value for each pixel is plotted against position, creating a profile of the staining intensity for that individual fiber. Shown here is a typical intensity profile from a light fiber, demonstrating intense mitochondrial staining near the sarcolemma and very faint staining in the interior of the fiber. From this profile, two SS regions and two IM regions are defined, and an average intensity value is calculated for each of these defined ranges. Additionally, a total average intensity value is calculated across the entire fiber (from peak to peak), to represent the total mitochondrial density of that fiber. (Fiber dimensions in (a) correspond to values on the $\mathrm{x}$-axis in (b).) 
pixel of the image crossed by the line. These values corresponded to the relative SDH-staining intensity, which is indicative of mitochondrial density. To determine the relative difference in mitochondrial density between the SS and IM regions of the fiber, intensity profile data were first exported to Microsoft Excel and plotted against position in the fiber (Fig. 1b). Two SS and two IM regions were then defined from these plots, and the average intensity value was calculated for each region. An SS region was defined as the portion of the line between the peak of the intensity curve and the point where the slope first becomes zero, while each IM region was demarcated as half of the portion of the line between flanking SS regions. The two SS and two IM intensity values in each radial profile were averaged together providing one final SS and IM value per fiber/subdivision. In addition, a total average intensity value was calculated across the entire diameter of the fiber/ subdivision to reflect total relative mitochondrial density. For each species, mitochondrial distribution and density measurements were determined from $n=24-56$ light fibers and $n=49-58$ dark fiber subdivisions.

Calculation of nuclear number volume and myonuclear domain

Animals $(N=3$ per species) were injected with $100 \mu$ of $1 \mathrm{mg} / \mathrm{ml}$ AlexaFluor 488-labeled wheat germ agglutinin (WGA; W11261; Molecular Probes). Injections were given straight into the hemolymph through the arthroidial membrane between the carapace and the coxa of the 5th pereiopod. WGA is a lectin that binds to glycoproteins on the basement membrane of the fiber sarcolemma (Wright 1984) and is used here to delineate fiber and subdivision boundaries. After injection, animals were provoked with a stick while in a container filled with filtered seawater (FSW) to elicit exercise and stimulate blood flow. Animals were then rested for 10 min in FSW and killed. The basal muscles were removed and fixed for $4-8 \mathrm{~h}$ in $4 \%$ paraformaldehyde in FSW, washed overnight in $25 \%$ sucrose, then flash frozen in liquid nitrogen. Frozen sections were cut on a Reichert-Jung Leica Cryocut 1800 microtome at $20 \mu \mathrm{m}$ thickness in a systematic random sampling method to ensure complete representation of nuclear distribution throughout the muscle (Howard and Reed 1998). Frozen sections were picked up on room temperature Superfrost PLUS slides and rinsed in PBS. Sections were incubated for $15 \mathrm{~min}$ in $300 \mathrm{nM}$ of blue-fluorescent probe $4^{\prime}, 6$-diamidino-2-phenylindole (DAPI; D1306; Molecular Probes) to label nuclei and then rinsed again for $3 \mathrm{~min}$ in PBS and mounted in 9:1 Tris/glycerol (0.1 M Tris, $\mathrm{pH}$ 7.4) mounting media. All images were taken with the Olympus FV1000 confocal microscope as single optical slices and included the $404 \mathrm{~nm}$ (DAPI), $488 \mathrm{~nm}$ (WGA) and differential interference contrast (DIC) channels. Images were viewed with Olympus Fluoview v. 1.6a software, and nuclei inside of each complete fiber cross section were counted and tallied as either SS, if they were in contact with the sarcolemma, or IM, if they were not in direct contact with the sarcolemma. Intrafiber SS nuclei can be difficult to distinguish from nuclei in cells in the extracellular space and in adjoining fibers. Information from the WGA and DIC channels allowed us to more accurately determine which peripherally located nuclei were truly inside the fiber. Fiber and subdivision cross-sectional area (CSA), circumference and mean diameter, as well as nuclear CSA, diameter (from fiber cross sections) and length (from longitudinal sections) were calculated as earlier.

As described previously (Hardy et al. 2009), the myonuclear domain (volume of cytoplasm per myonucleus) was calculated according to the formula used in Schmalbruch and Hellhammer (1977), and the nuclear number volume (number of nuclei per volume of cytoplasm) was calculated as the inverse of the myonuclear domain for SS and IM nuclei, respectively. For each species, estimates of nuclear number volume and domain were determined from $n=69$ 100 light fibers and $n=61-94$ dark fiber subdivisions.

Isolation and sequencing of $16 \mathrm{~S}$ ribosomal DNA

DNA extraction, amplification and sequencing protocols followed Mantellato et al. (2007) and Robles et al. (2007). Total genomic DNA was isolated from muscle tissue in the chelipeds of $O$. ocellatus and in the walking legs of C. magister using a DNeasy Kit (Qiagen, Inc., Valencia, CA). These were the only two species included in this study for which a partial $16 \mathrm{~S}$ rDNA sequence was not available on GenBank. Partial fragments of the 16 S ribosomal region of mtDNA (16S rDNA) were amplified by a polymerase chain reaction (PCR) on a PTC-100 thermal cycler (MJ Research). Each PCR reaction was performed in $50 \mu \mathrm{l}$ volumes containing $2 \mu \mathrm{l}$ of DNA template $(\sim 150 \mathrm{ng})$, $25 \mu \mathrm{l} \mathrm{GoTaq}{ }^{\circledR}$ Colorless Master Mix (Promega, Madison, WI), $21 \mu \mathrm{l}$ nuclease-free $\mathrm{H}_{2} \mathrm{O}$ and $1 \mu \mathrm{l}$ each of forward and reverse primer $(50 \mu \mathrm{M})$. Our thermal profiles were carried out as follows: initial denaturation cycle for $10 \mathrm{~min}$ at $95^{\circ} \mathrm{C}$, followed by 42 cycles of $1 \mathrm{~min}$ at $95^{\circ} \mathrm{C}, 1 \mathrm{~min}$ at $46^{\circ} \mathrm{C}$ and $2 \mathrm{~min}$ at $72^{\circ} \mathrm{C}$, with a final extension of $72^{\circ} \mathrm{C}$ for $10 \mathrm{~min}$. For these reactions, we used the forward primer 16Sar (5'-CGC CTG TTT ATC AAA AAC AT-3') paired with the reverse primer $16 \mathrm{Sbr}$ (5'-CCG GTC TGA ACT CAG ATC ACG T-3'), and the forward primer 16SL2 (5'-TGC CTG TTT ATC AAA AAC AT-3') paired with the reverse primer $1472\left(5^{\prime}\right.$-AGA TAG AAA CCA ACC TGG-3') (for references on primers see Palumbi et al. 1991; Schubart et al. 2000; Fratini et al. 2005). Both primer pairs produced clear, single bands of approximately 560-bp 
on a $1.2 \%$ agarose gel with ethidium bromide (Invitrogen, Corp., Carlsbad, CA). The resulting PCR products were purified (QIAquick PCR Purification Kit, Qiagen, Inc.) and sequenced on an ABI PRISM 3100 Genetic Analyzer using the ABI Big Dye Terminator Cycle Sequencing Kit v3.1. Consensus sequences for O. ocellatus (GenBank Accession no. FJ716615) and C. magister (FJ829795) were assembled using Sequencher v4.8 (Gene Codes, Corp., Ann Arbor, MI) and are available online.

Tree construction

The 16S rDNA sequences obtained above for $O$. ocellatus and $C$. magister were aligned with sequences for $16 \mathrm{~S}$ from C. sapidus (Gen Bank Accession no. AJ298189), P. sayi (DQ388053), P. spinimanus (DQ388056), P. gibbesii (DQ388057), C. maenas (DQ079709) and M. mercenaria (U20749) using ClustalX v2.0.10 (Larkin et al. 2007). A phylogeny was constructed with the beta version of MEGA v4.1 (Kumar et al. 2008), and a congruent topology was inferred by both maximum parsimony (MP) and neighborjoining (NJ) analyses. MP analysis was performed as a heuristic search with random sequence addition and all sites, including gaps, were equally weighted. A maximum likelihood model of NJ analysis was performed with pairwise deletion of gaps. Bootstrapping for both MP and NJ analyses used 1,000 replicates, and tree branches with confidence values $>50 \%$ were condensed.

Statistical and phylogenetic independent contrast analyses

To test for phylogenetic signal, we used Felsenstein's (1985) method of phylogenetically independent contrasts (PIC). The analysis was conducted with the Phenotypic Diversity Analysis Program (PDAP; Midford et al. 2005) and the subset package PDTREE (Garland et al. 1999; Garland and Ives 2000). The topology and branch lengths generated by the MP analysis of $16 \mathrm{~S}$ rDNA sequences were used in the PIC analysis, and a diagnostic test (plot between the absolute value of each standardized contrast and the standard deviation showed no relationship) found these branch lengths to be statistically acceptable after logtransformation (Garland et al. 1992). Standardized phylogenetic contrasts were calculated from log-transformed branch lengths and regressions (through the origin) were determined between pairs of standardized contrasts. The absence of a significant relationship between the contrasts (denoted by a slope that did not significantly differ from 0 ) was evidence of a phylogenetic signal in those data. Linear regressions were fit to the data (raw and contrasts) based on the ordinary least square (OLS) model and significance was accepted at $P<0.05$.
Student's $t$-tests were used to make pairwise comparisons between species. Given the number of comparisons, tests were Bonferroni corrected to maintain an overall $\alpha$ level of 0.05; differences between species were considered statistically significant if $P<0.0064$. All data are presented as means \pm SEM.

\section{Results}

Phylogenetic analysis

We generated a phylogeny of identical topology and highly similar branch lengths using MP and NJ analyses of $16 \mathrm{~S}$ rDNA sequences (Fig. 2). Based on this phylogeny, $C$. maenas and $O$. ocellates are likely not portunids, as they group more closely with the xanthids and the cancrids. In addition, C. magister (F. Cancridae) and M. mercenaria (F. Xanthidae) were more closely related to the Portunidae than $C$. maenas and $O$. ocellatus in a $16 \mathrm{~S}$ rDNA phylogeny that was rooted with the Caribbean spiny lobster, Panulirus argus (data not shown).

Animal body mass and fiber size

The adult animals used in this study ranged in size from 2.5 to $857.4 \mathrm{~g}$. The mean body mass per species was given as follows: $4.0 \pm 0.12 \mathrm{~g}$ (P. sayi), $10.17 \pm 0.08 \mathrm{~g}$ (P. gibbesii), $\quad 20.58 \pm 0.42 \mathrm{~g} \quad(P$. spinimanus $), \quad 37.98 \pm 0.61 \mathrm{~g}$ (C. maenas), $57.69 \pm 1.59$ (O. ocellatus), $163.05 \pm 3.18 \mathrm{~g}$ (C. sapidus), $359.53 \pm 3.8 \mathrm{~g}$ (M. mercenaria) and $778.04 \pm$ $4.23 \mathrm{~g}$ ( $C$. magister). Figure 3 demonstrates the variability in fiber structure that existed among a subset of the species examined in this study. Across all eight species, cross-sectional diameter of the anaerobic light fibers ranged from 73.89 to $1088.56 \mu \mathrm{m}$. Average light fiber diameter was larger in species with higher body mass $\left(r^{2}=0.91\right.$; $P=0.0002$ ) (Fig. 4a), and this relationship appeared to be generally independent of swimming ability (Fig. 4c). O.ocellatus and C. maenas were the only two species that did not significantly differ in mean light fiber diameter. This differed from the subdivisions of the aerobic dark fibers, which had no relationship between mean cross-sectional diameter and body mass ( $r^{2}=0.25 ; P=0.2122$ ) (Fig. 4b), but were significantly smaller in species possessing the ability to swim well (Fig. 4d). Those species pairs whose subdivision diameters did not significantly differ were $M$. mercenaria and $C$. magister, $C$. sapidus and $P$. sayi, and $P$. spinimanus and $P$. gibbesii. Dark fiber subdivision diameter ranged from 15.13 to $486.00 \mu \mathrm{m}$ across the eight species examined. A comparison of the phylogenetically standardized contrasts revealed that light fiber diameter was still significantly correlated with body mass $(P<0.001)$ 


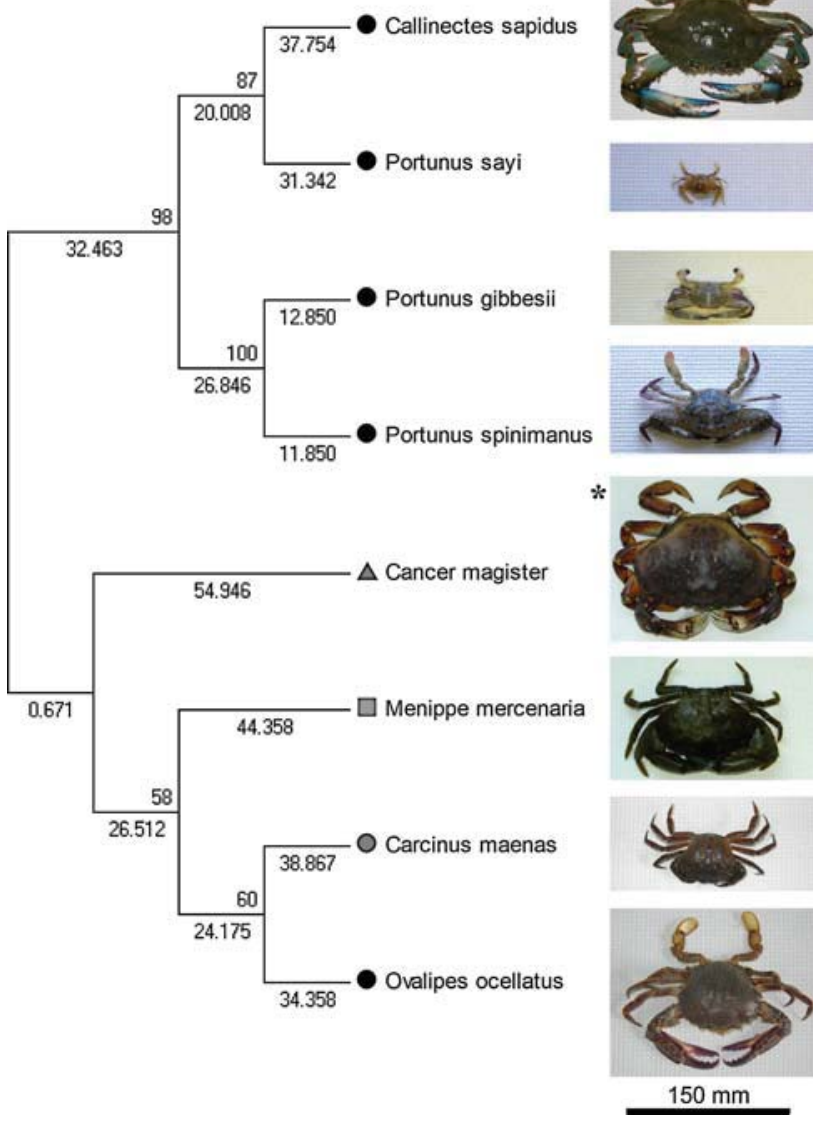

Fig. 2 Phylogenetic relationship among several brachyuran species [family Portunidae (circles), Xanthidae (square) and Cancridae (triangle)] based on 16S rDNA sequences. A congruent topology was inferred by maximum parsimony and neighbor-joining analyses. Values above each line are bootstrap values (1,000 replicates) and values below are branch lengths in number of mutations per time, as obtained from the MP analysis. Species are broadly classified as either excellent swimmers (black) or non-swimmers (gray) (Hartnoll 1971). Note that $C$. maenas and $O$. ocellatus, which are considered to be in the family Portunidae, along with the genera Portunus and Callinectes, appear to be more closely related to the family Xanthidae member, M. mercenaria. The * by C. magister indicates that the scale bar for this species is $300 \mathrm{~mm}$, instead of $150 \mathrm{~mm}$

(Fig. 4a-inset), while subdivision diameter remained independent of body mass $(P=0.564)$ (Fig. $4 \mathrm{~b}$-inset).

Mitochondrial and nuclear distribution

The relative distribution of mitochondria between the SS and IM regions of a fiber can be described in terms of the ratio of SS mitochondrial density to IM mitochondrial density (SS:IM intensity), as determined from the average intensity of the SDH staining. Using this approach, we measured a SS:IM average intensity of 7.4 in the light fibers of $C$. sapidus, which is consistent with a previous SS:IM calculation of 7.5 obtained using transmission electron microscopy and standard stereological measurements of mitochondrial volume density in the same muscle (Hardy et al. 2009). This indicates that the SDH-staining method accurately reflects relative mitochondrial distribution. A significant relationship between SS:IM intensity and fiber/ subdivision diameter indicates fiber size-dependant differences in the intracellular placement of mitochondria. Across all eight species, we observed that the SS:IM intensity was higher in both light fibers $\left(r^{2}=0.24, P<0.001\right)$ and dark fiber subdivisions $\left(r^{2}=0.50, P<0.001\right)$ with larger diameters (Fig. 5a). Thus, there is a higher density of SS mitochondria relative to IM mitochondria in larger fibers/ subdivisions. Figure 5b shows the relationship between the standardized contrasts for log-transformed SS:IM intensity and log-transformed diameter. The significant positive relationship found for both fiber types in the raw data was still present between the contrasts of the light fibers $(P=0.025)$, though not the dark fibers $(P=0.255)$.

The relative distribution of nuclei can be examined in a similar way. By examining the ratio between the SS nuclear number volume and the IM nuclear number volume (SSNV:IMNV), it is possible to assess whether nuclear position is different in fibers/subdivisions of varying sizes. We observed a significant negative relationship between SSNV:IMNV and diameter in both the light fibers $\left(r^{2}=0.55 ; P<0.0001\right)$ and the dark fiber subdivisions $\left(r^{2}=0.28 ; P<0.001\right)$ (Fig. 5c). This relationship indicates that there is a lower relative density of SS nuclei than IM nuclei per volume of cytoplasm in larger fibers. When the phylogenetically standardized contrasts were correlated, there was still a significant negative relationship between SSNV:IMNV and diameter in the light fibers $(P=0.006)$, but no longer in the dark fibers $(P=0.074)$ (Fig. 5d).

Mitochondrial and nuclear density

We evaluated the mass-specific scaling of mitochondrial density (from the total average SDH-staining intensity) using the following standard scaling equation: mitochondrial density $=a M^{b}$, where $a$ is a coefficient, $M$ is body mass and $b$ is the mass-specific scaling exponent. The $b$ values for the light and dark fibers based on total average intensity values (Fig. 6) were consistent with $b$ values from C. sapidus muscle for citrate synthase activity and mitochondrial volume density, which are standard measures of total aerobic capacity (Hardy et al. 2009; Johnson et al. 2004), again indicating that the SDH method effectively represented relative mitochondrial density.

The total average SDH intensity was lower in larger fibers over the range of sizes encompassing both the light fibers and dark fiber subdivisions (Fig. 7a). The dark fiber subdivisions had a strong negative relationship between 

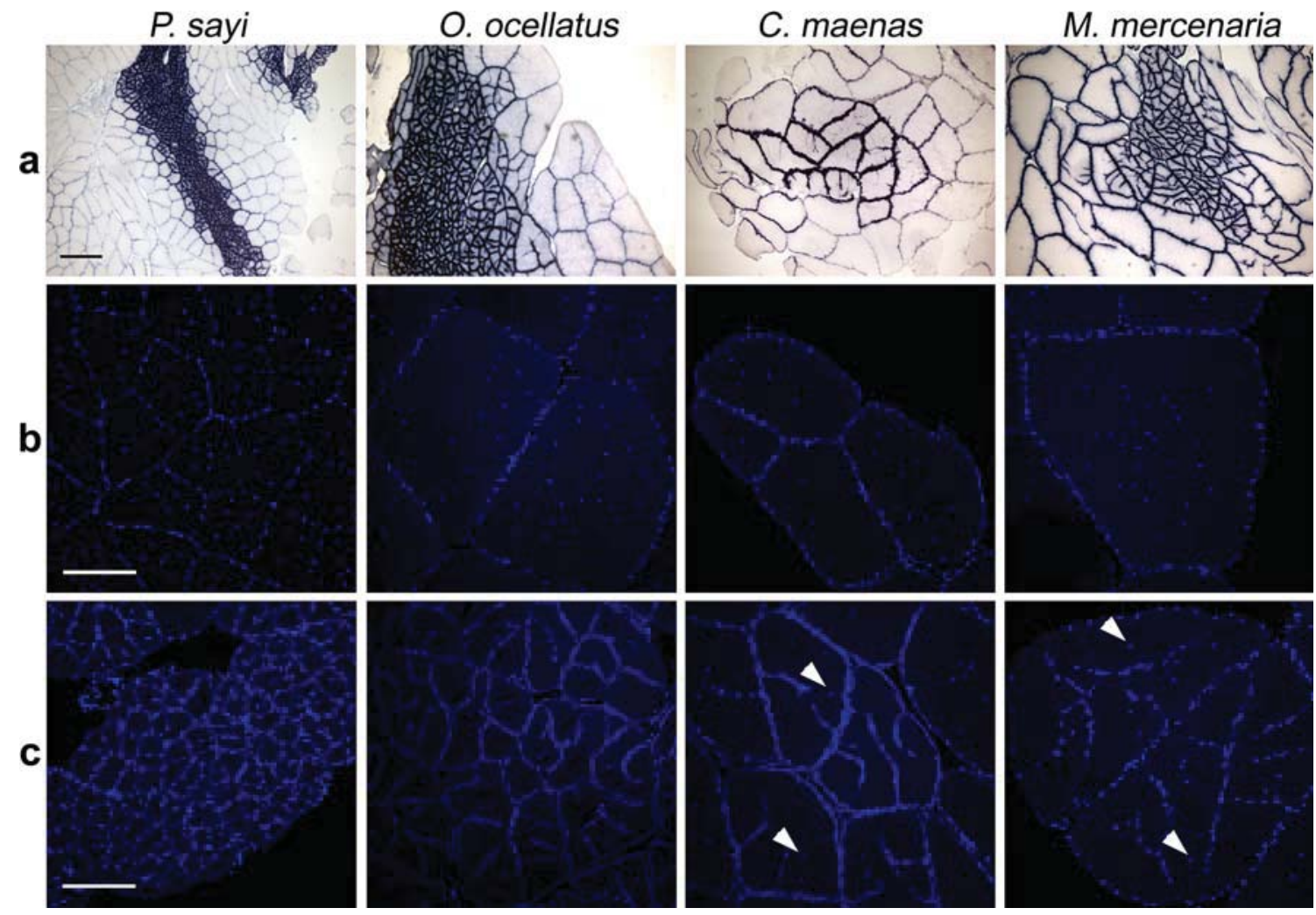

Fig. 3 Representative images of muscle cross sections stained for mitochondria with SDH (a) and nuclei with DAPI (b, c). These images demonstrate the variability in light fiber diameter and dark fiber subdivision diameter that exists among the species examined in this study (Note that images from only a subsample of the species examined are shown.). a In the SDH stained muscle, aerobic fibers are characterized by very dense staining, while the adjacent anaerobic light fibers stain much less intensely. This contrast reflects the large difference in mitochondrial density typical of these two fiber types. b Nuclear staining in the anaerobic light fibers. These images demonstrate an increase in the total number of intermyofibrillar nuclei

total intensity and subdivision size $\left(r^{2}=0.60 ; P<0.001\right)$, while the light fibers showed a weaker, though still significant, negative relationship $\left(r^{2}=0.06 ; P<0.001\right)$. Aerobic dark fibers are characterized by high mitochondrial densities ( $\sim 25 \%$ in C. sapidus; Johnson et al. 2004), while anaerobic light fibers have substantially lower mitochondrial densities ( $<1 \%$ in C. sapidus; Hardy et al. 2009). In combination, however, the dark and light fibers form a fairly continuous spectrum of sizes with decreasing total intensity values with fiber/subdivision diameter (Fig. 7a).

With respect to nuclei, the IMNV was independent of diameter for the light fibers $\left(r^{2}=0.0013 ; P=0.3015\right)$ (data not shown). (Since the small aerobic subdivisions frequently had no IM nuclei, the IMNV could not always be accurately calculated, and so no relationship to diameter could be provided for these fibers). These results contrast with the SSNV that was negatively related to diameter for both light fibers $\left(r^{2}=0.63 ; P<0.0001\right)$ and dark fiber

with fiber size, but a fairly conserved myonuclear domain (the volume of cytoplasm 'serviced' by a single nucleus) for each species, regardless of fiber size. c Nuclear staining in the aerobic dark fibers. Nuclei are located exclusively adjacent to the sarcolemma in the smaller subdivisions, but, as in the light fibers, nuclei begin to appear in the intermyofibrillar zone as subdivision diameter increases (arrowheads). Scale bar $500 \mu \mathrm{m}(\mathbf{a}), 150 \mu \mathrm{m}(\mathbf{b}, \mathbf{c})$ (Note that the resolution of Fig. 3 is reduced to meet the standards for on-line publication. A higher resolution version of Fig. 3 is available online in the Electronic Supplementary Material.)

subdivisions $\left(r^{2}=0.24 ; P<0.0001\right)$ (data not shown). Combined, the size effects on IMNV and SSNV resulted in a myonuclear domain that increased with diameter for both light fibers $\left(r^{2}=0.43 ; P<0.0001\right)$ and dark fibers $\left(r^{2}=0.20 ; P<0.0001\right)$ (Fig. 7b). That is, while the IMNV remained constant, the number of SS nuclei per volume of fiber/subdivision was lower in larger fibers, which meant that each nucleus was responsible for servicing a larger volume of cytoplasm.

Figure 7c shows the relationship between myonuclear domain and total aerobic capacity, as indicated by total average intensity value from SDH staining, for all eight species. We demonstrated that in each species except for one (O. ocellatus), the anaerobic light fibers, which are characterized by a much lower aerobic capacity than the aerobic dark fibers, exhibit smaller myonuclear domains. Only $O$. ocellatus and $P$. sayi did not have significantly different myonuclear domains between the light and dark fibers. 
(a)
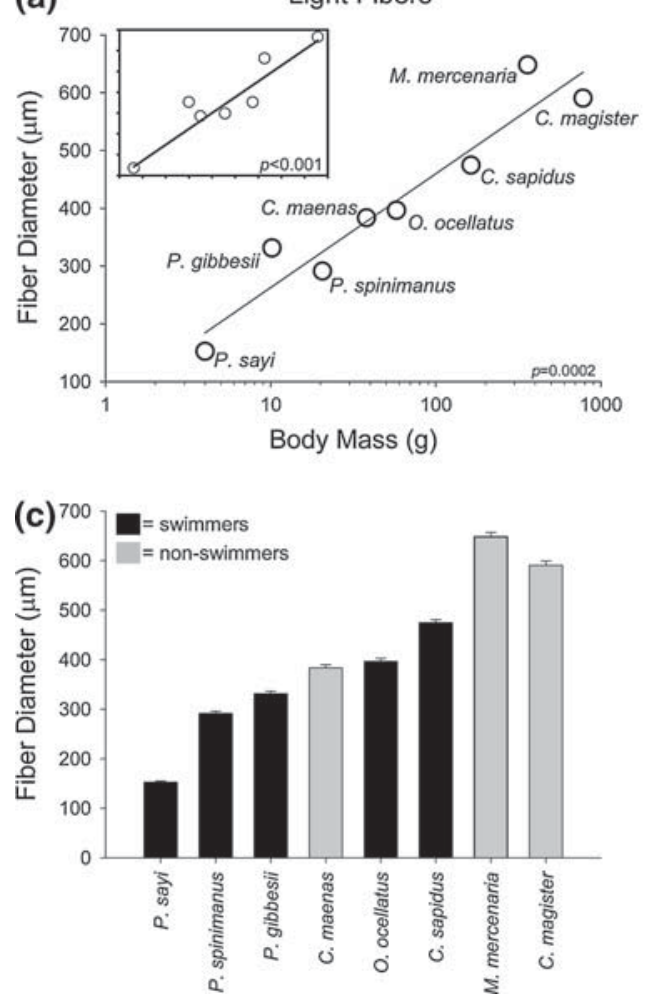

Fig. 4 Fiber and subdivision sizes in the anaerobic light fibers (left panels) and aerobic dark fibers (right panels). Scatterplot of the mean cross-sectional diameter of the light fibers (a) and dark fiber subdivisions (b) plotted as a function of body mass. Insets show the relationship between the phylogenetically standardized contrast values for diameter and log-transformed body mass. In each case, they describe the same relationship as the raw data. Mean crosssectional diameter of the anaerobic light fibers (c) and aerobic dark

\section{Discussion}

Species of brachyuran crabs in the family Portunidae are characterized by their exceptional swimming abilities. The blue crab (C. sapidus), for example, has been shown to swim at peak burst speeds up to $0.5 \mathrm{~m} / \mathrm{s}$ and maintain sustained speeds of $\sim 0.1-0.2 \mathrm{~m} / \mathrm{s}$ during migratory swims (Carr et al. 2004; Zimmer-Faust et al. 1994). The evolution of swimming in the portunids has been facilitated by a number of morphological adaptations to their 5 th pereiopods, carapace and basal swimming musculature (Hartnoll 1971).

Based on our 16S rDNA phylogeny (Fig. 2), Carcinus maenas and Ovalipes ocellatus may be historically misplaced in the family Portunidae and are likely more closely related to the Families Xanthidae (M. mercenaria) and Cancridae (C. magister). This is consistent with other recent molecular phylogenies, which characterized Ovalipes as the most distant genera within the Portunidae (in a 16S rDNA based phylogeny exclusively of portunids; Mantelatto et al. 2007) and grouped Carcinus with the Cancridae and not Portunidae (in a multi-family phylogeny
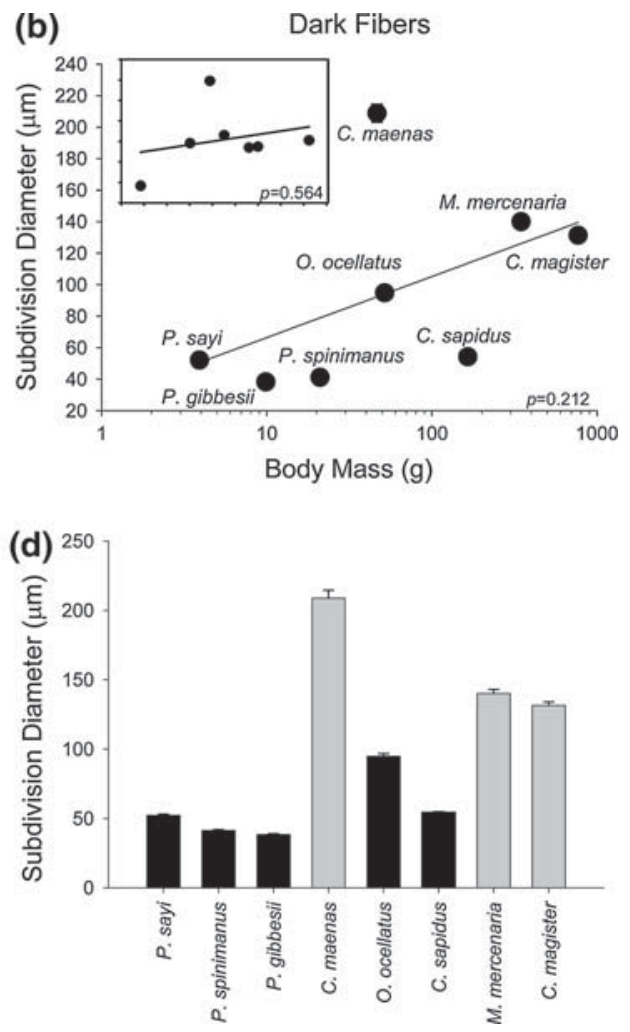

fiber subdivisions (d) for each species. Species are arranged in order of increasing adult body mass and are categorized as either excellent swimmers (black) or non-swimmers (gray). Sample sizes were $n=195-242$ light fibers per species and $n=222-488$ dark fiber subdivisions per species. Values shown are means \pm SEM (error bars in $\mathbf{a}$ and $\mathbf{b}$ are smaller than the symbols). See text for additional details

of brachyurans based on the gene for arginine kinase; Mahon and Neigel 2008). Differences in basic body shape and swimming ability would also suggest that $O$. ocellatus and $C$. maenas are more closely related to the xanthid and cancrid crabs. They both lack the prominent anterolateral spines that typify most portunids and share a more oval/ hexagonally shaped carapace that is more characteristic of xanthids and cancrids. Furthermore, the 5th pereiopods in C. maenas resemble unmodified walking legs, not oar-like paddles, and this species has an extremely limited swimming capacity. However, the closely related $O$. ocellatus exhibits the same flat, broadened 5th pereiopods as the other portunids and is also a proficient swimmer (though not as good as members of Callinectes and Portunus) (personal observation).

Our phylogeny suggests that swimming ability and some of the morphological modifications associated with swimming have evolved multiple times within the brachyurans: at least once by the portunids, as we classify them here, and once within the genus Ovalipes, which may not be monophyletic with the portunids. Sustained swimming is 

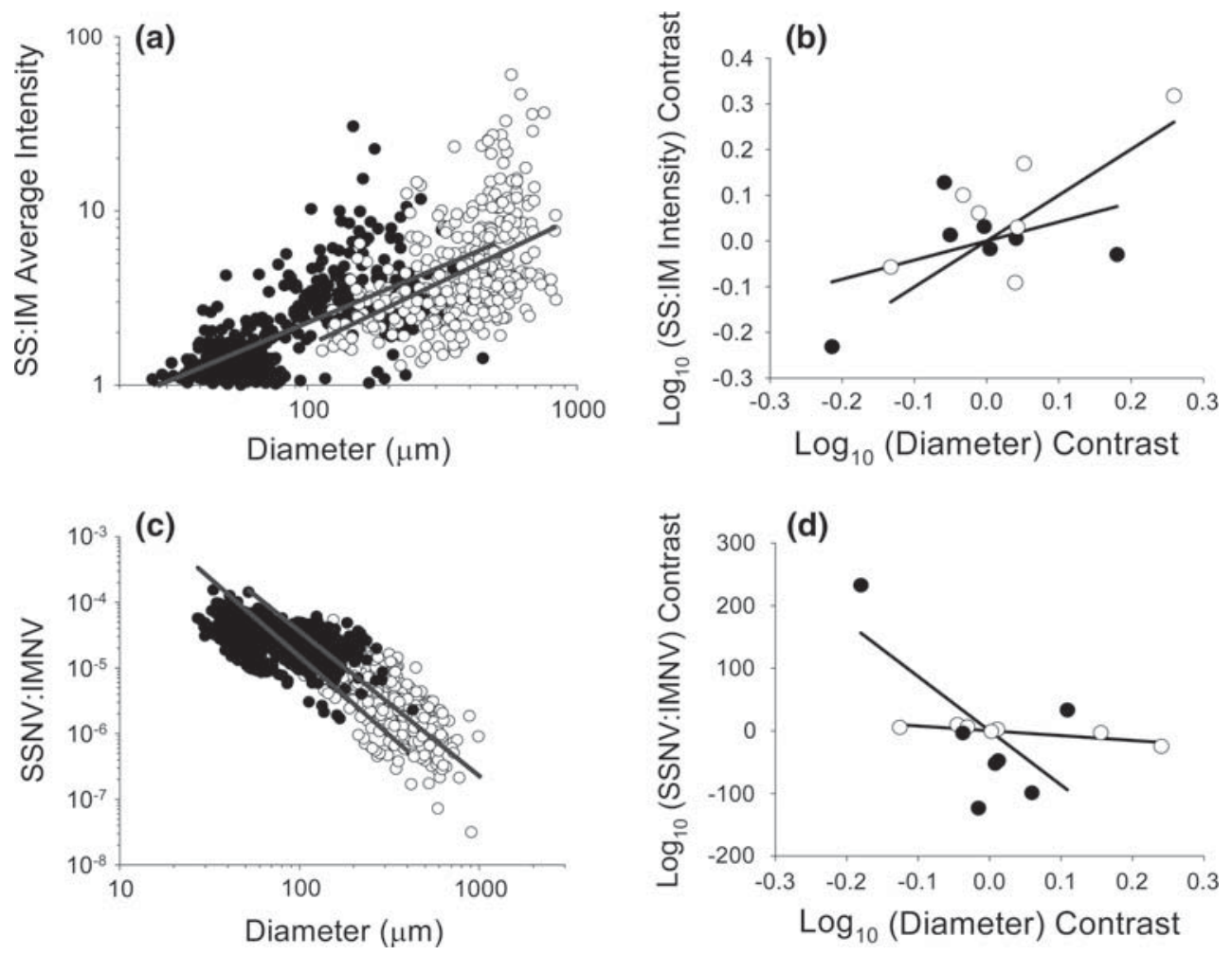

Fig. 5 Differences in mitochondrial and nuclear distribution with size for anaerobic light fibers (open circle) and aerobic dark fiber subdivisions (filled circle). a Scatterplot of raw data for the ratio of SS to IM average intensity value (SS:IM intensity) and diameter. The SS:IM intensity increases with diameter in both the light fibers and the dark fibers. This reflects an increase in density of SS mitochondria and a decrease in density of IM mitochondria that occur as fibers get bigger. b Standardized independent contrast values of log-transformed SS:IM intensity plotted as a function of log-transformed diameter. The significant positive relationship observed in the raw

aerobically powered by the dark fibers and requires high rates of ATP turnover, which necessitates short intracellular diffusion distances and hence a smaller diameter (Crow and Kushmerick 1982; Kushmerick et al. 1992). How then is sustained aerobic swimming activity supported in the relatively large dark fibers and do all swimming crabs solve this problem in the same way?

In $C$. sapidus, the dark fibers have evolved a network of highly perfused, mitochondria-rich subdivisions (Hardy et al. 2009; Tse et al. 1983) that increase in number, but maintain a constant size $(\sim 35 \mu \mathrm{m})$ during fiber growth (Johnson et al. 2004). The perfused subdivisions result in greatly reduced diffusion distances and increased oxygen flux to the mitochondria. In this way, the aerobic fibers preserve an effectively small metabolic diameter throughout development. Reaction-diffusion mathematical models have demonstrated that the high rate of ATP turnover required of steady-state swimming activity in the blue crab dark fibers could not be supported at the large sizes that would exist if they were not subdivided $(\sim 300 \mu \mathrm{m}$ diffusion

data disappears in the dark fibers, but persists in the light fibers. c Scatterplot of raw data for SSNV:IMNV and diameter. The SSNV:IMNV decreases with diameter in both the light fibers and the dark fiber subdivisions. This negative relationship results from a decrease in the SSNV and a constant IMNV with size (data not shown). d Standardized independent contrast values of log-transformed SSNV:IMNV plotted as a function of log-transformed diameter. The negative relationship observed in the raw data disappears in the dark fibers, but persists in the light fibers

distance) (Hardy et al. 2009; Kinsey et al. 2007). However, the same high reaction rate could be achieved over the short diffusion distances characteristic of the smaller subdivisions (Johnson et al. 2004). Thus, subdividing the dark fibers appears to be essential to the evolution of sustained swimming behavior in C. sapidus.

In the current study, we observed that the dark fibers were highly subdivided in all of the swimming species that we examined ( $P$. sayi, $P$. spinimanus, $P$. gibbesii, $O$. ocellatus and $C$. sapidus) (see Fig. 2 for representative examples). It therefore appears that all swimming crabs have evolved the ability to support high levels of sustained aerobic activity in the same way, by dividing their dark fibers into metabolically smaller functional units, while the contractile functional unit appears to be the entire fiber (see Hardy et al. 2009). Presumably, these aerobic dark fibers have secondarily evolved from anaerobic light fiber precursors, which in crustaceans can have very large diameters $(>600 \mu \mathrm{m}$ ) (Boyle et al. 2003; Hardy et al. 2009; Jimenez et al. 2008). This notion is supported by the essentially 


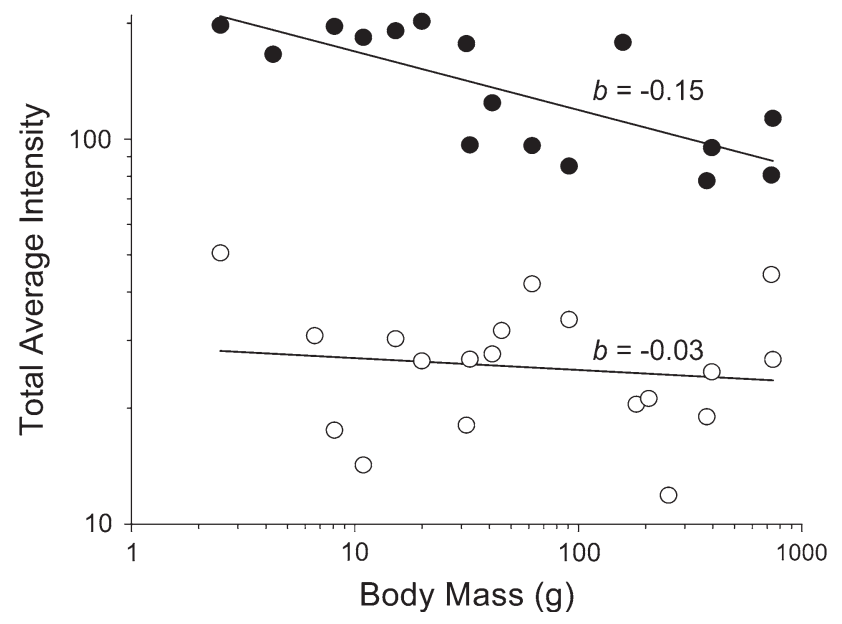

Fig. 6 Relationship between total average SDH-staining intensity and body mass for anaerobic light fibers (open circle) and aerobic dark fiber subdivisions (filled circle). This graph shows the massspecific metabolic scaling of aerobic capacity in each fiber type. The regression equation for the dark fibers is total intensity $=2.38 \mathrm{M}^{-0.15}$ $\left(r^{2}=0.547, P=0.001 ; M\right.$ is body mass $)$ and for the white fibers total intensity $=1.46 \mathrm{M}^{-0.03}\left(r^{2}=0.018, P=0.58\right)$. See text for additional details

non-subdivided morphology of aerobic fibers in C. maenas, a species that cannot swim, compared to the highly subdivided fibers in the closely related $O$. ocellatus, a species well adapted for swimming.

Previous modeling has shown that further increases in diameter or ATP turnover rate would result in substantial diffusion constraints to aerobic metabolic processes in C. sapidus (Hardy et al. 2006; Kinsey et al. 2007). If the dark fibers evolved from the large anaerobic light fiber precursors, then the development of subdivisions resulted from a unidirectional pressure to be smaller as sustained swimming behavior evolved. Thus, dark fiber subdivisions are likely only as small as they need to be to support the rate of ATP turnover required by aerobic swimming, but no smaller. Accordingly, we found that species with efficient swimming abilities had smaller average subdivision sizes than species that could not swim (Fig. 4b, d). This was true even for $O$. ocellatus, which appears to have independently evolved the ability to swim. However, it is interesting that subdivisions in $O$. ocellatus, while relatively small, are still significantly larger on average than the other swimming portunids $(P$. sayi, $P$. gibbesii, $P$. spinimanus and C. sapidus). This may reflect the fact that $O$. ocellatus lacks some of the morphological adaptations for swimming that are apparent in the other swimming crabs (e.g., a modified carapace shape). Therefore, they may not be able to attain the sustained swimming velocities characteristic of C. sapidus and the genus Portunus. If this is the case, ATP turnover demands would likely be lower, and selective
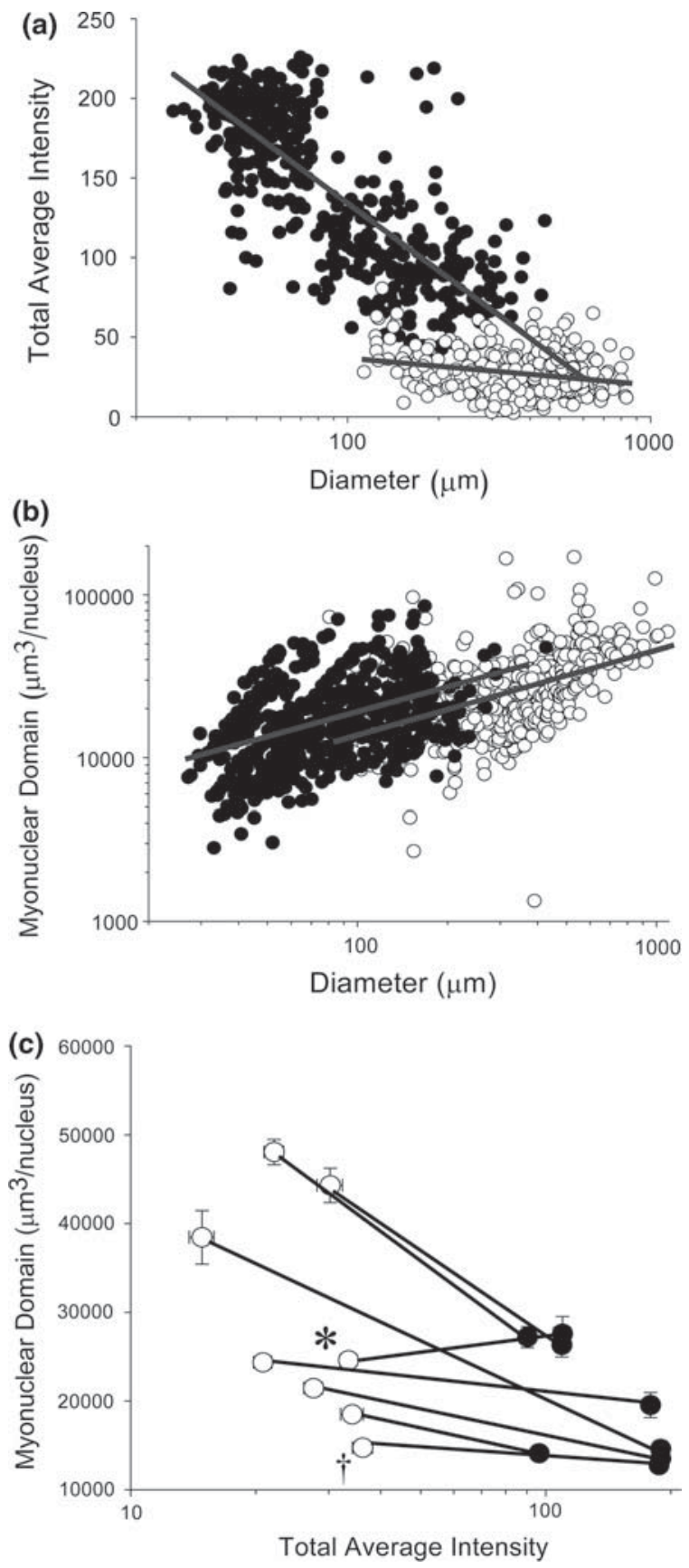

Fig. 7 Differences in mitochondrial density (as determined from total average SDH intensity) (a) and myonuclear domain (b) with size, as well as the relationship between mitochondrial density and myonuclear domain (c) for anaerobic light fibers (open circle) and aerobic dark fiber subdivisions (filled circle). a Total intensity of SDH staining decreases with diameter in both light fibers and dark fiber subdivisions. b Myonuclear domain increases with diameter in both the light fibers and the dark fiber subdivisions, which results from a decrease in SSNV and a constant IMNV with size (data not shown). c Relationship between myonuclear domain and total average intensity in anaerobic light fibers and aerobic dark fiber subdivisions. Black lines connect the light and dark fiber values from the same species and are for visualization purposes only. Thus, myonuclear domain is significantly higher in fibers with lower aerobic capacity (i.e., light fibers) in all species except for $O$. ocellatus $(*)$ and $P$. sayi $(\dagger)$ 
pressure for small subdivisions and high mitochondrial densities would be lessened.

The non-swimming species (C. maenas, $C$. magister and M. mercenaria) also possessed subdivided dark fibers (see Fig. 3), although subdivision of these fibers was far less extensive than in the other swimming species (Fig. 4d). These species are generally associated with the benthic environment and move by walking with a relatively low frequency of limb motion. This mode of locomotion requires a much lower rate of ATP turnover than sustained pelagic swimming, which is powered by a high contraction frequency. Hence, aerobic metabolic rates in the dark fibers of the non-swimming crabs can be supported with larger subdivisions. These differences are consistent with behavioral observations. In pilot experiments, we observed that C. maenas, when suspended in the water column of an aquarium, was only capable of sustaining continuous aerobic swimming for an average of $25 \mathrm{~min}$, while some of the swimming crabs (C. sapidus, P. spinimanus, $O$. ocellatus) were capable of continuously swimming for $>8 \mathrm{~h}$. It is interesting that the stone crab, M. mercenaria, has dark fibers that are somewhat more poised for aerobic function (i.e., moderately subdivided), despite the fact that these animals cannot swim. It is likely that the 5th pereiopods in these animals are used for holding on tightly to rocky substrates for sustained durations, although the ATP turnover rate required for this is clearly less than for swimming. Thus, evolutionary changes in fiber subdivision size may be shaped by other behavioral demands, such as sustained walking and gripping.

The light fibers, in contrast to dark fibers, power anaerobic burst swimming and only rely on maximal rates of aerobic metabolism during recovery from exercise (Curtin et al. 1997; Kushmerick and Paul 1976). However, aerobically fueled, post-contractile recovery requires much lower ATP turnover rates than steady-state swimming in the dark fibers. As a result of the decreased metabolic demands, there is apparently little or no selective pressure on the diameter of the light fibers. Supporting this view was the direct relationship between light fiber diameter and body mass (Fig. 4a), which suggests that each species experiences relatively unconstrained hypertrophic growth during development. This multi-species relationship was independent of phylogeny and consistent with previous observations in $C$. sapidus, which undergo an ontogenetic increase in light fiber diameter with body mass (Boyle et al. 2003; Kinsey et al. 2005). Thus, within a single species and across many species, diffusion does not appear to limit light fiber diameter because the rates of aerobic ATP turnover are so low. However, at least in C. sapidus, this is only the case because the mitochondria undergo a dramatic shift in distribution as the fibers get bigger. In small fibers from juveniles, mitochondria are uniformly distributed throughout the IM and SS regions of the cell, but mitochondria are found clustered almost exclusively at the sarcolemma in the large fibers from adults (Boyle et al. 2003; Hardy et al. 2009). Mitochondrial function is dependent on adequate diffusive flux of $\mathrm{O}_{2}$ from the blood and small metabolites like ATP to cytosolic ATPases. Thus, a shift in mitochondria toward a SS distribution during fiber growth reflects the need to minimize diffusion distances for $\mathrm{O}_{2}$, at the expense of larger diffusion distances for small metabolites. Hardy et al. (2009) used a reaction-diffusion mathematical model to demonstrate that the measured rate of ATP turnover in the adult (large) light fibers in $C$. sapidus can only be met when the mitochondria cluster at the sarcolemma, thus demonstrating the functional rationale for this ontogenetic reorganization (Hardy et al. 2009).

If the earlier interpretation is correct, then we would expect to see the same fiber-size-specific differences in mitochondrial distribution between small and large light fibers in the present study. In this case, however, the observed differences would not reflect ontogenetic changes in distribution, but rather would represent variations in the relative diffusion constraints in adult muscle of species with different maximum fiber sizes. We found that the SS:IM average SDH intensity ratio was higher in larger fibers indicating that there were more SS mitochondria relative to IM mitochondria as fiber size increased. We have now demonstrated in multiple species of crustaceans that mitochondrial distribution changes in response to diffusion constraints. Nyack et al. (2007) demonstrated that the same pattern exists during growth of fish white muscle. Furthermore, we have shown that the same fiber size-dependant distribution occurs in both the light fibers and the dark fibers, suggesting that this is an aspect of cellular design adopted by multiple muscle fiber types.

While the positive relationship between SS:IM intensity and diameter was significant in both the light and dark fibers in the raw (phylogenetically uncorrected) data (Fig. 5a), a comparison of the phylogenetically standardized contrasts revealed that the relationship was only independent of phylogeny in the light fibers (Fig. 5b). Thus, mitochondrial distribution in the light fibers is not strongly influenced by phylogenetic inertia; that is, it does not occur as a result of a shared common ancestry among these species. This finding further supports the notion that mitochondrial distribution is a plastic property that can change in response to prevailing diffusion conditions. In contrast, the absence of a relationship in the dark fibers indicates that a phylogenetic signal largely explains the distribution of mitochondria in the subdivisions.

Nuclei, unlike mitochondria, do not rely on oxygen directly, yet they (and their associated synthetic apparatus) 
are involved in the simultaneous transcription, translation and diffusive flux of a variety of molecules ranging in size from small metabolites to larger macromolecules, and potentially even membrane bound vesicles. In C. sapidus, nuclei had a pattern of redistribution during fiber growth that was the opposite of that seen for mitochondria (Hardy et al. 2009). In the smallest juvenile fibers, nuclei were located exclusively in the SS region of the cell, but as fiber size increased nuclei had SS, as well as an abundance of IM nuclei. A reaction-diffusion model revealed that without the size-dependent shift in nuclear distribution, relevant rates of transcription/translation would decrease by three orders of magnitude (Hardy et al. 2009). We demonstrated the same relationship between nuclear distribution and fiber size in both light and dark fibers in the eight species examined in this study. The SSNV:IMNV ratio was always lower in larger fibers, which reflected an increased number of IM nuclei relative to SS nuclei (Fig. 5c). Nuclei appear to solve the problem of long intracellular diffusion distances in larger fibers by shifting toward a more IM distribution. As seen for mitochondrial distribution, however, the relationship between nuclear distribution and diameter was significant in a comparison of the phylogenetically weighted contrasts only in the light fibers (Fig. 5d).

Why are differences in mitochondrial and nuclear distribution with fiber/subdivision size independent of phylogeny in the light fibers only? Since the rate of aerobic metabolism in the light fibers is relatively low, there does not seem to be a limit on fiber size and, as we have shown, size of the light fibers is not linked to phylogeny (rather it is strongly related to body mass; Fig. 4a). Thus, the PIC analysis was able to effectively disentangle the effect of shared ancestry from the effect of fiber size and demonstrate that cellular organelle distribution is independent of phylogeny in the light muscle. In contrast, subdivision size in dark fibers is highly constrained by aerobic demand-and variation in ATP demand is strongly linked to behavior (i.e., swimmers vs. non-swimmers/walkers). It is apparent in our phylogeny that the eight species we examined group strongly according to their mode of locomotion (see Fig. 2). Thus, if organelle distribution is, in fact, a direct consequence of subdivision size (and hence changing intracellular diffusion conditions), it will be intrinsically linked to phylogeny even if it is not the result of shared ancestry. Perhaps the most compelling evidence that organelle distribution in the dark fibers is actually not the consequence of phylogenetic inertia can be garnered directly from the light fibers. As we demonstrated, organelle distribution in the light fibers is independent of phylogeny, and it seems likely that the mechanism for regulating organelle distribution in adjacent skeletal muscle groups would be the same. In effect, the light fibers serve as an internal control for comparison based on their proximity to the dark fibers.
Figure 7 a demonstrates that mitochondrial density was inversely related to fiber or subdivision size. These data support the generally accepted relationship between fiber type, oxidative capacity and fiber size. Highly oxidative (aerobic) fibers have small diameters and high mitochondrial densities, compared to glycolytic (anaerobic) fibers, which are much larger in diameter and have very low mitochondrial densities. Like mitochondrial density, nuclear density also decreased with size. However, nuclei are typically characterized in terms of myonuclear domain, which is simply the inverse of nuclear number volume (a measurement of nuclear density) (Fig. 7b). In both the dark and light fibers, myonuclear domain was larger in fibers/ subdivisions with an increased diameter. Similar increases in domain with fiber size have been reported during hypertrophic growth in mammalian skeletal muscle (Bruusgaard et al. 2005; Cheek et al. 1971; Giddings and Gonyea 1992).

Myonuclear domain was also negatively related to aerobic capacity. Domain size was significantly higher in the light fibers than the dark fiber for each species, excluding $P$. sayi and $O$. ocellatus (Fig. 7c). This is not completely surprising given that protein synthesis rates are generally recognized to be higher in smaller slow-twitch oxidative (aerobic) fibers than larger fast-twitch glycolytic (anaerobic) fibers (Bates and Millward 1983; Garlick et al. 1989; Goldberg 1967; Kelly et al. 1984; Laurent et al. 1978). This finding is also consistent with the work of Bruusgaard et al. (2003) who suggested that fast glycolytic fibers are more stable than slow oxidative fibers, owing to much longer protein half-lives, which may explain why these authors observed nuclear numbers in glycolytic fibers that did not vary in proportion to volume as in the oxidative fibers, but rather varied in proportion to fiber surface area. A lower protein turnover rate would allow each nucleus to efficiently service a larger volume of cytoplasm and over potentially longer intracellular diffusion distances.

It is generally proposed that nuclear numbers increase during hypertrophy in skeletal muscle fibers to maintain a somewhat constant myonuclear domain size (Bruusgaard et al. 2003, 2006; Cheek et al. 1971; Giddings and Gonyea 1992; Jaspers et al. 2006; Roy et al. 1999). However, we propose that it is the maximum diffusion distance within that domain that needs to be kept small. Nuclear domain can be conserved during growth by increasing nuclear density only at the fiber periphery or by increasing nuclear density both in the SS region and in the IM region. During hypertrophic growth in fibers of $C$. sapidus myonuclear domain is conserved by the latter approach (Hardy et al. 2009), and Figs. $3 \mathrm{a}, \mathrm{b}$ and $5 \mathrm{c}, \mathrm{d}$ indicate that the same pattern exists across multiple species. While the myonuclear domain size is higher in larger fibers/subdivisions (Fig. 7b), the increased IMNV means that intracellular diffusion distances for large 
proteins, and mRNA molecules will be much smaller than they would be if all nuclei were restricted to the fiber periphery. Accordingly, we found that the mean distance between any two myonuclei was fairly constant across species and between fiber types (regardless of fiber diameter and nuclear distribution), ranging from $23.5 \pm 0.34$ to $36.3 \pm 0.58 \mu \mathrm{m}$ in light fibers and $20.7 \pm 0.73$ to $29.5 \pm 0.58 \mu \mathrm{m}$ in the dark fibers, which is consistent with nuclear spacing in mouse skeletal muscle with an exclusively SS distribution (Bruusgaard et al. 2003).

In summary, the present study demonstrated that the high aerobic demand associated with sustained swimming required the evolution of subdivided aerobic dark fibers that promote intrafiber perfusion and short diffusion distances for oxygen and small metabolites. The aerobic muscle from the species with the greatest capacity for sustained swimming had the smallest subdivisions and the highest mitochondrial densities. The anaerobic light fibers have much lower rates of aerobic ATP demand and are not subdivided. In larger fibers/subdivisions, the mitochondria are distributed closer to the sarcolemmal membrane, placing them closer to the source of oxygen. In contrast, the nuclei are located at the sarcolemmal membrane in small fibers/subdivisions, but are distributed more uniformly in larger fibers/subdivisions, preserving short diffusion distances for nuclear substrates and products. These findings are consistent with prior experimental data and reaction-diffusion mathematical models that evaluated ontogenetic changes in fiber size and muscle aerobic structure in $C$. sapidus swimming muscle. In C. sapidus, the observed rate of aerobic ATP turnover in both dark and light fibers would not be possible in larger fibers without these structural modifications. The present study demonstrated that these responses to cellular diffusion constraints are broadly observed in brachyuran crabs and are independent of phylogeny, suggesting that they are key design features associated with the evolution of swimming behavior.

Acknowledgments The authors are grateful for the helpful comments of Drs. Richard Dillaman, Ann Pabst, Richard Satterlie and Robert Roer, as well as the technical assistance of Mark Gay and Dr. Marcel van Tuinen. This research was supported by a National Science Foundation grant to STK (IOS-0719123) and a National Institute of Arthritis and Musculoskeletal and Skin Diseases grant to STK (R15-AR052708).

\section{References}

Badrinath AS, White AG (2003) Contrasting patterns of mitochondrial redistribution in the early lineages of Caenorhabditis elegans and Acrobeloides sp. PS1146. Dev Biol 258:70-75

Bates PC, Millward DJ (1983) Myofibrillar protein turnover. Synthesis rates of myofibrillar and sarcoplasmic protein fractions in different muscles and the changes observed during postnatal development and in response to feeding. Biochem J 214:587-592 Bitoun M, Maugenre S, Jeannet P-Y, Lacene E, Ferrer X, Laforet P, Martin J-J, Laporte J, Lochmuller H, Beggs AH, Fardeau M, Eymard B, Romero NB, Guicheney P (2005) Mutations in dynamin 2 cause dominant centronuclear myopathy. Nat Genet 37:1207-1209

Blomberg SP, Garland T (2002) Tempo and mode in evolution: phylogenetic inertia, adaptation and comparative methods. J Evol Biol 15:899-910

Boyle KL, Dillaman RM, Kinsey ST (2003) Mitochondrial distribution and glycogen dynamics suggest diffusion constraints in muscle fibers of the blue crab, Callinectes sapidus. J Exp Zool 297A:1-16

Bruusgaard JC, Liestøl K, Ekmark M, Kollstad K, Gunderson K (2003) Number and spatial distribution of nuclei in the muscle fibers of normal mice studied in vivo. J Physiol 551(2):467478

Bruusgaard JC, Brack AS, Hughes SM, Gunderson K (2005) Muscle hypertrophy induces by the ski protein: cyto-architecture and ultrastructure. Acta Physiol Scand 185:141-149

Bruusgaard JC, Liestøl K, Gunderson K (2006) Distribution of myonuclei and microtubules in live muscle fibers of young, middle-aged, and old mice. J Appl Physiol 100:2024-2030

Carr SD, Tankersley RA, Hench JL, Forward RB Jr, Luettich RA Jr (2004) Movement patterns and trajectories of ovigerous blue crabs Callinectes sapidus during the spawning migration. Estuar Coast Shelf Sci 60:567-579

Cheek DB, Holt AB, Hill DE, Talbert JL (1971) Skeletal muscle cell mass and growth: the concept of the deoxyribonucleic acid unit. Pediatr Res 5:312-328

Chilibeck PD, Syrotuik DG, Bell GJ (2002) The effect of concurrent endurance and strength training on quantitative estimates of subsarcolemmal and intermyofibrillar mitochondria. Int J Sports Med 23(1):33-39

Cochran DM (1935) The skeletal musculature of the blue crab Callinectes sapidus Rathbun. Smithson Misc Collns 92:1-96

Crow MT, Kushmerick MJ (1982) Chemical energetics of slow- and fast-twitch muscle of the mouse. J Gen Physiol 79:147-166

Curtin NA, Kushmerick MJ, Wiseman RW, Woledge RC (1997) Recovery after contraction of white muscle fibres from the dogfish Scyliorhinus canicula. J Exp Biol 200:1061-1071

Felsenstein J (1985) Phylogenies and the comparative method. Am Nat 125:1-15

Fiedler RA (1930) Solving the question of crab migration. Fish Gazette 47:18-21

Fratini S, Vannini M, Cannicci S, Schubart CD (2005) Tree-climbing mangrove crabs, a case of convergent evolution. Evol Ecol Res 7:219-233

Frederick RL, Shaw JM (2007) Moving mitochondria: establishing distribution of an essential organelle. Traffic 8:1668-1675

Fusco D, Bertrand E, Singer RH (2004) Imaging of single mRNAs in the cytoplasm of living cells. Prog Mol Subcell Biol 35:135-150

Garland T Jr, Ives AR (2000) Using the past to predict the present: confidence intervals or regression equations in phylogenetic comparative methods. Am Nat 155:346-364

Garland T, Harvey PH, Ives AR (1992) Procedures for the analysis of comparative data using phylogenetically independent contrasts. Syst Biol 41:18-32

Garland T Jr, Midford PE, Ives AR (1999) An introduction to phylogenetically based statistical methods, with a new method for confidence intervals on ancestral states. Am Zool 39:374388

Garlick PJ, Maltin CA, Baillie AG, Delday MI, Grubb DA (1989) Fiber-type composition of nine rat muscles. II. Relationship to protein turnover. Am J Physiol 257:E828-E832 
Giddings CJ, Gonyea WJ (1992) Morphological observations supporting muscle fiber hyperplasia following weight-lifting exercise in cats. Anat Rec 233:178-195

Goldberg AL (1967) Protein synthesis in tonic and phasic skeletal muscle. Nat Lond 216:1219-1220

Hardy KM, Locke BR, Da Silva MD, Kinsey ST (2006) A reactiondiffusion analysis of energetics in large muscle fibers secondarily evolved for aerobic locomotor function. J Exp Biol 209:3610-3620

Hardy KM, Dillaman RM, Locke BR, Kinsey ST (2009) A skeletal muscle model of extreme hypertrophic growth reveals the influence of diffusion on cellular design. Am J Physiol Regul Integr Comp Physiol 296:R1855-R1867

Hartnoll RG (1971) The occurrence, methods and significance of swimming in the Brachyura. Anim Behav 19:34-50

Hoppeler H, Vogt M (2001) Muscle tissue adaptations to hypoxia. J Exp Biol 204:3133-3139

Howald H, Hoppeler H, Claassen H, Mathieu O, Straub R (1985) Influences of endurance training on the ultrastructural composition of the different muscle fiber types in humans. Pflugers Arch 403(4):369-376

Howard CV, Reed MG (1998) Unbiased stereology, 3-dimensional measurements in microscopy. BIOS Scientific, Oxford

Jaspers RT, Feenstra HM, van Beek-Harmsen BJ, Huijing PA, van der Laarse WJ (2006) Differential effects of muscle fibre length and insulin on muscle-specific mRNA content in isolated mature muscle fibres during long-term culture. Cell Tissue Res 326:795-808

Jimenez AG, Locke BR, Kinsey ST (2008) The influence of oxygen and high-energy phosphate diffusion on metabolic scaling in three species of tail-flipping crustaceans. J Exp Biol 211:3214-3225

Johnson LK, Dillaman RM, Gay DM, Blum JE, Kinsey ST (2004) Metabolic influences of fiber size in aerobic and anaerobic muscles of the blue crab, Callinectes sapidus. J Exp Biol 207:4045-4056

Judy MH, Dudley DL (1970) Movement of tagged blue crabs in North Carolina waters. Commer Fish Rev 32:29-35

Kayar SR, Claassen H, Hoppeler H, Weibel ER (1986) Mitochondrial distribution in relation to changes in muscle metabolism in rat soleus. Respir Physiol 64(1):1-11

Kelly FJ, Lewis SE, Anderson P, Goldspink DF (1984) Pre- and postnatal growth and protein turnover in four muscles of the rat. Muscle Nerve 7:235-242

Kim SK, Yu SH, Jeong-Hwa S, Hübner H, Buchholz R (1998) Calculations on $\mathrm{O}_{2}$ transfer in capsules with animal cells for the determination of maximum capsule size without $\mathrm{O}_{2}$ limitation. Biotech Lett 20:549-552

Kinsey ST, Pathi P, Hardy KM, Jordan A, Locke BR (2005) Does intracellular metabolite diffusion limit post-contractile recovery in burst locomotor muscle? J Exp Biol 208:2641-2652

Kinsey ST, Hardy KM, Locke BR (2007) The long and winding road: influences of intracellular metabolite diffusion on cellular organization and metabolism in skeletal muscle. J Exp Biol 210:3505-3512

Koch L (1996) What size should a bacterium be? A question of scale. Annu Rev Microbiol 50:317-348

Kumar S, Dudley J, Nei M, Tamura K (2008) MEGA: a biologistcentric software for evolutionary analysis of DNA and protein sequences. Brief Bioinfom 9:299-306

Kushmerick MJ, Paul RJ (1976) Aerobic recovery metabolism following a single isometric tetanus in frog sartorius muscle at $0^{\circ} \mathrm{C}$. J Physiol 254:693-709

Kushmerick MJ, Meyer RA, Brown TR (1992) Regulation of oxygen consumption in fast- and slow-twitch muscle. Am J Physiol 263:C598-C606 (Cell Physiol 32)

Larkin MA, Blackshields G, Brown NP, Chenna R, McGettigan PA, McWilliam H, Valentin F, Wallace IM, Wilm A, Lopez R,
Thompson JD, Gibson TJ, Higgins DG (2007) Clustal W and Clustal X version 2.0. Bioinformatics 23:2947-2948

Laurent GJ, Sparrow MP, Bates PC, Millward DJ (1978) Turnover of muscle proteins in the fowl (Gallus domesticus). Rates of protein synthesis in fast and slow skeletal, cardiac and smooth muscle of the adult fowl. Biochem J 176:393-401

Mahon BC, Neigel JE (2008) Utility of arginine kinase for resolution of phylogenetic relationships among brachyuran genera and families. Mol Phylogenet Evol 48:718-727

Mainwood GW, Rakusan K (1982) A model for intracellular energy transport. Can J Physiol Pharmacol 60:98-102

Mantelatto FL, Robles R, Felder DL (2007) Molecular phylogeny of the western Atlantic species of the genus Portunus (Crustacea, Brachyura, Portunidae). Zool J Linn Soc 150:211-220

Midford PE, Garland T Jr, Maddison WP (2005) PDAP package of Mesquite. Version 1.07

Milner DJ, Weitzer G, Tran D, Bradley A, Capetanaki Y (1996) Disruption of muscle architecture and myocardial degeneration in mice lacking desmin. J Cell Biol 134:1255-1270

Nyack AC, Locke BR, Valencia A, Dillaman RM, Kinsey ST (2007) Scaling of postcontractile phosphocreatine recovery in fish white muscle: effect of intracellular diffusion. Am J Physiol Regul Integr Comp Physiol 292:R2077-R2088

Palumbi S, Martin A, Romano S, McMillan WO, Stice L, Grabowski G (1991) The simple fool's guide to PCR. Department of Zoology and Kewalo Marine Laboratory, University of Hawaii, Honolulu

Presnell JK, Schreibman MP (1997) Animal tissue techniques, 5th edn. Johns Hopkins University Press, Baltimore

Ralston E, Lu Z, Biscocho N, Soumaka E, Mavrodis M, Prats C, Lomo T, Capetanaki Y, Plous T (2006) Blood vessels and desmin control the positioning of nuclei in skeletal muscle fibers. J Cell Physiol 209(3):874-882

Rathbun MJ (1930) The cancroid crabs of America of the families euryalidae, portunidae, atelecyclidae, cancridae, and xanthidae. Bull US Natl Mus 152:1-609

Robles R, Schubart CD, Conde JE, Carmona-Suarez C, Alvarez F, Villalobos JL, Felder DL (2007) Molecular phylogeny of the American Callinectes Stimpson, 1860 (Brachyura: Portunidae), based on two partial mitochondrial genes. Mar Biol 150:12651274

Roy RR, Monke SR, Allen DL, Edgerton VR (1999) Modulation of myonuclear number in functionally overloaded and exercised rat plantaris fibers. J Appl Physiol 87:634-642

Rube DA, van der Bliek AM (2004) Mitochondrial morphology is varied and dynamic. Mol Cell Biochem 256-257:331-339

Russell B, Dix DJ (1992) Mechanisms for intracellular distribution of mRNA: in situ hybridization studies in muscle. Am J Physiol 262:C1-C8 (Cell Physiol 31)

Russell B, Motlagh D, Ashley WW (2000) Form follows function: how muscle shape is regulated by work. J Appl Physiol 88:11271132

Schmalbruch H, Hellhammer U (1977) The number of nuclei in adult rat muscles with special reference to satellite cells. Anat Rec 189:169-176

Schubart CD, Neigel JE, Felder DL (2000) Use of the mitochondrial $16 \mathrm{~S}$ rRNA gene for phylogenetic and population studies of Crustacea. Crustac Issues 12:817-830

Smirnova L, Shurland D-L, Ryazantsev SN, van der Bliek AM (1998) A human dynamin-related protein controls the distribution of mitochondria. J Cell Biol 143:351-358

Spirito CP (1972) An analysis of swimming behaviour in the Portunid crab Callinectes sapidus. Mar Behav Physiol 1:261-276

Starr DA (2007) Communication between the cytoskeleton and the nuclear envelope to position the nucleus. Mol BioSyst 3:583-589

Starr DA, Han M (2002) The role of ANC-1 in tethering nuclei to the actin cytoskeleton. Science 298:406-409 
Teissier G (1939) Biometrie de la cellule. Tabulae Biologicae 19:1-64

Thompson DW (1917) On growth and form. Cambridge University Press, Cambridge

Tse FW, Govind CK, Atwood HL (1983) Diverse fiber composition of swimming muscles in the blue crab, Callinectes sapidus. Can J Zool 61:52-59

Tyler S, Sidell BD (1984) Changes in mitochondrial distribution and diffusion distances in muscle of goldfish upon acclimation to cold temperatures. J Exp Biol 232:1-9

van Blerkom JV (1991) Microtubule mediation of cytoplasmic and nuclear maturation during the ear. Proc Natl Acad Sci 88:50315035
Williams A (1974) The swimming crabs of the genus Callinectes (Decapoda: Portunidae). Fish Bull 72:685-798

Wright CS (1984) Structural comparison of the two distinct sugar binding sites in wheat germ agglutinin isolectin II. J Mol Biol 178:91-104

Zimmer-Faust RK, Fielder DR, Heck KL, Coen LD, Morgan SG (1994) Effects of tethering on predatory escape by juvenile blue crabs. Mar Ecol Prog Ser 111:299-303 